\title{
Detection and Imaging of Magnetic Field in the Microwave Regime With a Combination of Magnetic Losses Material and Thermofluorescent Molecules
}

\author{
H. Ragazzo ${ }^{1}$, S. Faure ${ }^{2}$, J. Carrey ${ }^{2}$, F. Issac ${ }^{1}$, D. Prost ${ }^{1}$, and J. F. Bobo ${ }^{13}$ \\ ${ }^{1}$ DEMR, ONERA, Université de Toulouse, F-31055 Toulouse, France \\ ${ }^{2}$ LPCNO, INSA, 31077 Toulouse, France \\ ${ }^{3}$ CEMES, CNRS, 31055 Toulouse, France
}

\begin{abstract}
Characterization of the electromagnetic field emitted by various sources (antenna, radar, etc.) is an important issue, either for civil or defense applications. The measurement of electromagnetic field may be performed either by local probes or by thermography imaging, in particular with an infrared camera. The latter method, called electromagnetic infrared (EMIR) has been developed several years ago. We have recently successfully implemented this technique in the domain of visible light thanks to the combination of a film sensitive to either electric field (slightly conductive film) or magnetic field (insulating film with ferromagnetic particles) and a polymer coating doped with fluorescent molecules with an emission depending on temperature, indeed a thermofluorescent sensor. We present our recent results obtained imaging the microwave magnetic field emitted in the near field of a zeroth-order resonator (ZOR) antenna. The sensing film is a stack of FLEX-TOKIN magnetic absorber, already tested by EMIR infrared thermography in our group. A $20 \mu \mathrm{m}$ film composed of a mixture of rhodamine $\mathrm{B}(\mathrm{RhB})$ in an epoxy matrix is screen printed on the FLEX surface. RhB, in ethanol solution, has a thermofluorescence coefficient of $\sim 2 \% / \mathrm{K}$ at room temperature at its maximum of fluorescence of $595 \mathrm{~nm}$. Excitation light is delivered by an array of blue $470 \mathrm{~nm}$ LEDs. The 4.29 GHz microwave excitation of the ZOR device is amplitude modulated at $0.1-0.5 \mathrm{~Hz}$ in order to cancel any thermal drift and convection of the thermofluorescence image. Low-frequency excitation modulation also allows noise cancellation via image pixel demodulation postprocessing. We present the deduced temperature mapping of the sensing film placed $2 \mathrm{~mm}$ above the ZOR patch antenna.
\end{abstract}

Index Terms-Electromagnetism, fluorescence, magnetic losses, thermography.

\section{INTRODUCTION}

W E PRESENT, in this paper, a novel method for electromagnetic field measurement and imaging which is based on thermofluorescence. In a first step, we detail the principles of this technique; then, we present new results of near-field emission of an antenna. Finally, we will review the potential evolutions of our technique for its improvement.

Microwave technologies play an important role in many fields from public applications (phone, communications) to more specific (radar, civil and military aeronautics, medicine, etc.). In this context, an important issue is the study of interactions between microwaves and electric or electronic devices from a scientific and technical point of view. This is the so-called electromagnetic compatibility (EMC) domain. With the raise of society preoccupations concerning the multiplication of radio emitters for mobile communication applications, EMC is playing a large role. All of these facts motivate efforts for the development of new electromagnetic field sensors able to give a quick environment imaging.

Our work is based on electromagnetic infrared (EMIR) technology [1] developed, and patented by ONERA. We propose, here, a novel method using thermofluorescence: the incident electric or magnetic field to be measured heats a thin foil covered with fluorescent molecules (fluorophore) with

Manuscript received June 5, 2018; revised July 10, 2018; accepted July 21, 2018. Corresponding author: J. F. Bobo (e-mail: jfbobo@cemes.fr).

Color versions of one or more of the figures in this paper are available online at http://ieeexplore.ieee.org.

Digital Object Identifier 10.1109/TMAG.2018.2860520 a fluorescence intensity depending on the amplitude of the incident field. This allows imaging in the visible light domain contrary to infrared emission used in EMIR technique. The thermofluorescent film needs to be excited with appropriate light for fluorescence. We have recently patented this new method [2] and named it electromagnetic visible imaging (EMVI).

The interest of this technique is, like EMIR, to be able to visualize and eventually to quantify the amplitude of a magnetic or electric field by a relatively non-intrusive way on the surface of a thin film that absorbs one part of the incident wave: a 2-D cartography of the field can thus be obtained on a relatively large surface (several $\mathrm{dm}^{2}$ ), in a few seconds (much faster than a local probe that would be moved step by step to scan the whole surface). The main advantages of EMVI over EMIR are, on one hand, the reduced cost of visible cameras and optics compared to infrared, and on the other hand, the possibility to get local measurements with the combination of an optical fiber and a thermofluorescent sensor for example for biological applications.

\section{Presentation of EMVI Technique}

\section{A. Development of Films With Electric or Magnetic Losses}

As in EMIR method, we use polymer foils with small conductivity for electric field imaging, or with ferromagnetic losses for magnetic film imaging. Such foils are subsequently covered by a polymer film containing fluorescent molecules as presented in Section III. 


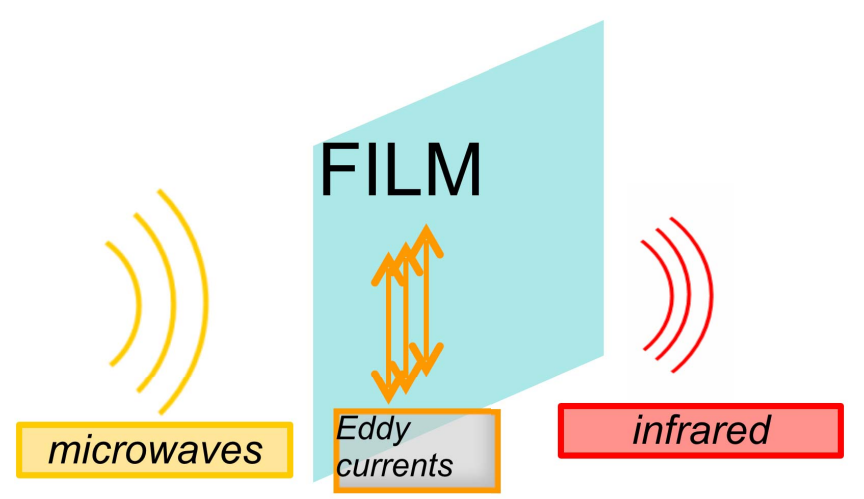

Fig. 1. Schematics of an incident electromagnetic wave on a conductive film.

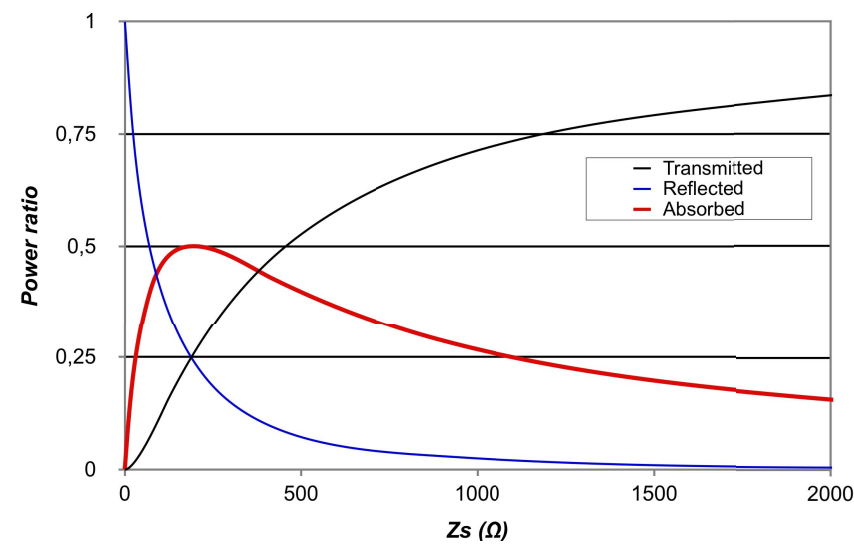

Fig. 2. Transmitted, absorbed, and reflected power for an incident electromagnetic plane wave on a thin plane as a function of its surface impedance $Z_{S}$.

For electric field imaging, the best sensing film is Kapton doped with graphite with a sheet resistivity $Z_{S}=\rho / t$ ( $\rho$ is the foil resistivity and $t$ is the thickness) larger than $1000 \Omega$ in order to get a low reflectivity of the incoming electromagnetic wave (see Fig. 1 for a schematics). Fig. 2 shows the transmitted, reflected, and absorbed powers for an incident plane wave, larger film sheet resistance yields low reflection of the electromagnetic wave with significant absorption.

Concerning magnetic field component, the sensing film has to be insulating with high permeability elements (patterned ferromagnetic film as already published [3], [4] or commercial FLEX-TOKIN absorber composed of ferromagnetic micro pellets in a polymer matrix) [5]. Depending on the frequency domain of the electromagnetic emission, two distinct processes can occur. At relatively low frequencies (1-100 kHz), hysteresis losses will cause heating with an effective absorption defined by the specific absorption rate $(\mathrm{SAR}) . \mathrm{SAR}=A \cdot f$, where $A$ is the area of the magnetization hysteresis loop (in $\mathrm{J} / \mathrm{g}$ ) and $f$ is the excitation frequency. Reference [6] for a detailed review of this mechanism. In the microwave regime, ferromagnetic resonance (FMR) losses or precessional losses will take place. They are related to the effective magnetic losses thickness defined by $t_{L}=\mu^{\prime} \cdot t$. ( $\mu^{\prime \prime}$ is the imaginary part of the permeability of the ferromagnetic material and $t$ is the effective thickness.) Fig. 3 illustrates magnetic
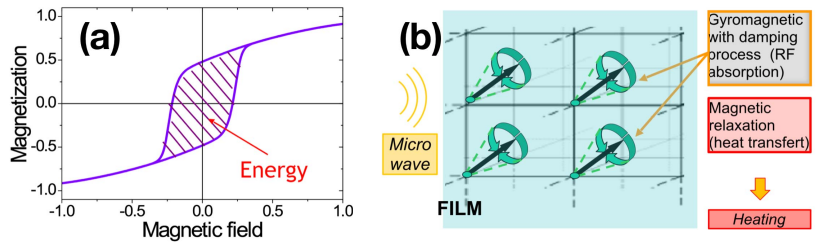

Fig. 3. Sketches of (a) low-frequency (kilohertz) magnetic losses process due to hysteresis losses and (b) microwave (gigahertz) FMR losses.

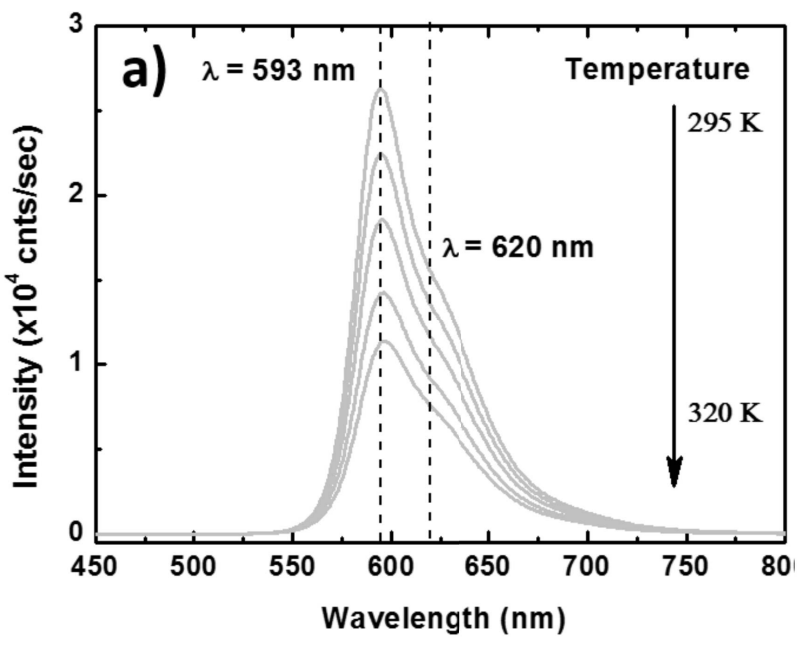

Fig. 4. Fluorescence spectra for a solution of $\mathrm{RhB}$ in ethanol as a function of temperature.

loss processes. In our previous papers, we have shown that ferromagnetic losses in the microwave range can cause significant heating up to $2 \mathrm{~K}$ for a moderate excitation in a TEM line.

\section{B. Development of Thermofluorescent Films}

Fluorescence is caused by the radiative recombination of excited states in fluorophore molecules. Such compounds can absorb incident light at a given wavelength and rapidly reemit fluorescence at a different (larger) wavelength. Once the incident photon is absorbed, the molecule is in an $S^{*}$ excited state. Internal relaxation from $S^{*}$ to $S_{0}$ (fluorescence singlet state) takes place prior to $S_{0}$ radiative transition. A large number of fluorophores are available with various properties (absorption wavelength, emission wavelength, thermal behavior, etc.). We have chosen rhodamine $\mathrm{B}(\mathrm{RhB})$ that is soluble in ethanol (solvent) and cellulose acetate or epoxy (polymers). Excitation wavelength is $470 \mathrm{~nm}$ and fluorescence occurs at 550-650 nm. Therefore, our films are exposed to $470 \mathrm{~nm}$ blue light emitted by a LED array. Absorption quantum yield of $\mathrm{RhB}$ is $70 \%$. Fig. 4 shows the temperature dependence of $\mathrm{RhB}$ fluorescence around room temperature. The thermofluorescence coefficient is approximately $-2 \% / \mathrm{K}$, making $\mathrm{RhB}$ a good candidate for thermofluorescence imaging. Therefore, we have spin-coated Kapton-graphite or FLEX-TOKIN foils with RhB-cellulose acetate or screen-printed RhB-epoxy for EMVI experiments. Typical fluorescent film thickness is $10-30 \mu \mathrm{m}$. 
Electromagnetic

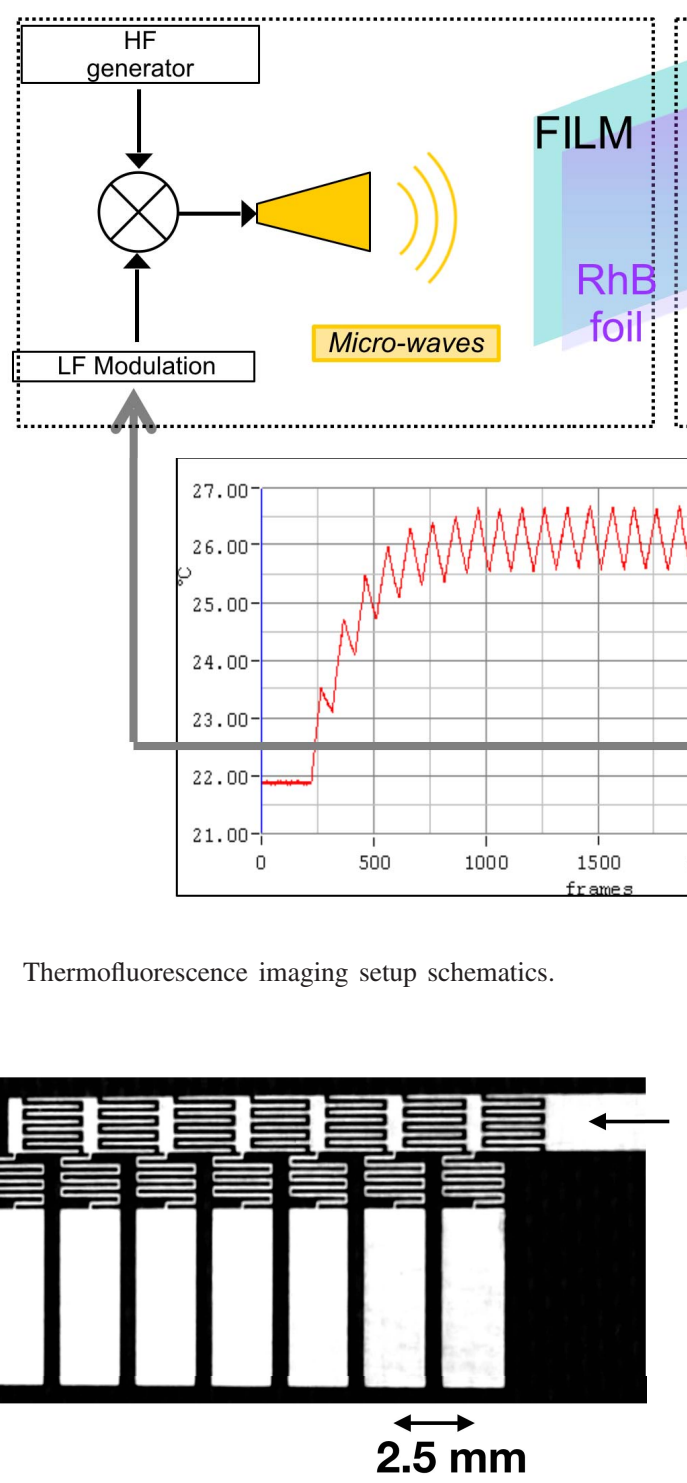

Fig. 6. ZOR antenna image. Each emitting element is $2.5 \mathrm{~mm}$ wide.

\section{Thermofluorescence Setup for Electromagnetic Field Imaging}

Fig. 5 represents the schematics of our EMVI setup. A microwave emitter is sending time-modulated $(0.1-0.5 \mathrm{~Hz})$ excitation to the sensing film. This film is also exposed to a $470 \mathrm{~nm}$ blue light LED source. Film heating is simultaneously imaged with a infrared camera (EMIR) and an S-CMOS camera equipped with a low-pass $520 \mathrm{~nm}$ filter to collect RhB fluorescence. A computer controls image collection and microwave intensity modulation between 0.1 and $0.5 \mathrm{~Hz}$ typically. This amplitude modulation helps for cancellation of all continuous thermal effects like natural convection or thermal drifts. It also allows, after Fourier image demodulation, to increase the signal-to-noise ratio of thermofluorescence imaging.

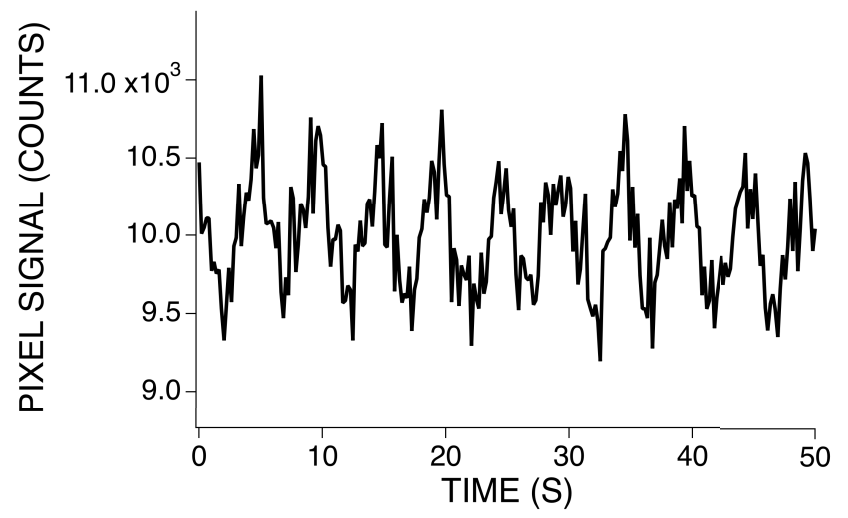

Fig. 7. Time dependence of the intensity of one pixel during microwave modulation at $0.2 \mathrm{~Hz}$ and $37 \mathrm{dBm}$ on ZOR antenna.

\section{Imaging ExAmple With a SPECIFIC PATCH ANTENNA}

We present, here, some of our best thermography images of the electric and magnetic field emitted by a zeroth-order resonator (ZOR) antenna [7]. This antenna has a narrow bandwidth centered at $4.29 \mathrm{GHz}$ and is composed of a periodical set of emitting elements (see Fig. 6).

Respectively, a $30 \mu \mathrm{m}$ Kapton-graphite and a $25 \mu \mathrm{m}$ thick FLEX film are placed $2 \mathrm{~mm}$ over the ZOR antenna. These films are covered with a $20 \mu \mathrm{m}$ RhB-epoxy fluorescent film. LED array provides a $470 \mathrm{~nm}$ excitation of several hundred $\mu \mathrm{W} / \mathrm{cm}^{2}$ up to few $\mathrm{mW} / \mathrm{cm}^{2}$. Dhyana400D 4 Mpixel 

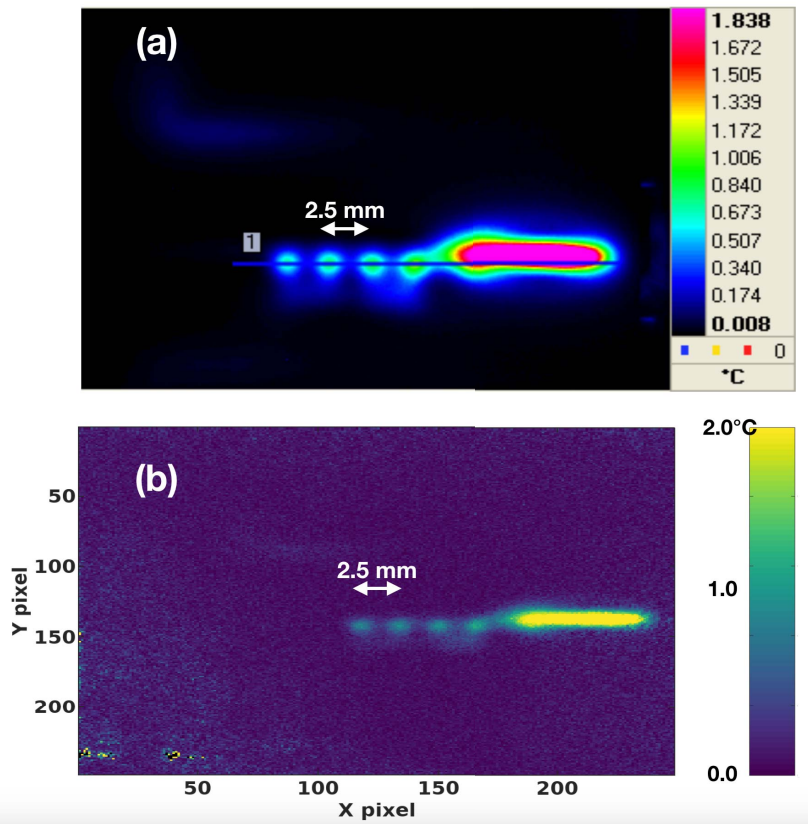

Fig. 8. (a) EMIR and (b) EMVI temperature imaging of ZOR antenna magnetic field emission. RF modulated input is $39 \mathrm{dBm}(8 \mathrm{~W})$. Line labeled 1 is used for image profiles presented in Fig. 9.

S-CMOS camera and CEDIP infrared camera (as a reference) are placed in front of the device.

After image collection, pixel per pixel data treatment is done for both kinds of images (visible and infrared). Fig. 7 shows a typical time dependence of S-CMOS camera pixel intensity during microwave modulation. Demodulation of the whole images collected with magnetic sensing film is shown in Fig. 8 which presents ZOR antenna emitted magnetic field heating by Fig. 8(a) EMIR and Fig. 8(b) EMVI methods. The deduced RF magnetic field is proportional to the square root of heating. From reference EMIR measurements performed on the base plane of a TEM line with similar FLEX film and RF excitation, we could extract a maximum value of the microwave magnetic field emitted by the ZOR antenna of $8 \mathrm{~A} / \mathrm{m}$ in near field. EMIR image reveals a main emission lobe with a $+1.9{ }^{\circ} \mathrm{C}$ heating and secondary lobes $\left(\Delta T=+0.5^{\circ} / 0.7^{\circ}\right)$. EMVI method yields quite similar result, despite with slightly more noise. In order to check the good agreement between the two imaging techniques, we have plotted in Fig. 9 the $\Delta T$ heating profiles along ZOR antenna axis. Image profiles along the main lobe collected in infrared and visible methods are very similar and validate EMVI technique. EMVI heating profiles are plotted for various microwave powers ranging from 33 to $39 \mathrm{dBm}$ ( 2 to $8 \mathrm{~W}$ ) showing the linearity and detection threshold of the present setup that we estimate to $\sim 0.1{ }^{\circ} \mathrm{C}$. Note that this is the first observation, up to our knowledge, of the magnetic field emitted by a microwave antenna in the visible light domain by thermofluorescence.

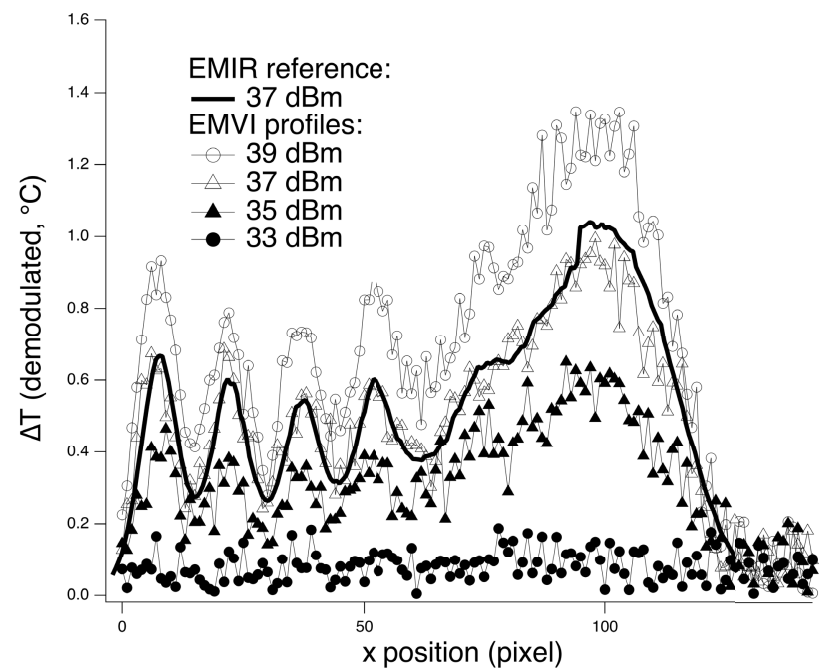

Fig. 9. Image intensity profiles along line 1 (see Fig. 8) of EMIR reference thermic image and several EMVI measurements for increasing RF input powers from 33 to $37 \mathrm{dBm}(2$ to $8 \mathrm{~W})$.

\section{CONCLUSION}

This new EMVI method gives encouraging results. Systematic implementation work is presently being done in order to improve all steps of this technique (fluorophore and resin combinations, film coating process, image demodulation, and selection of the wavelength of light excitation for fluorescence). EMVI is proposed as a new thermooptic imaging sensor for EMC.

\section{ACKNOWLEDGMENT}

This work was supported in part by the NEXT Laboratory of Excellence, CNRS, Université de Toulouse and in part by CNRS Interdisciplinary Mission DEFINSTRUM.

\section{REFERENCES}

[1] P. Levesque and L. Leylekian, "Capteur vectoriel de champs électromagnétiques par thermographie infrarouge," French Patent 9816079 A, Dec. 17, 1998.

[2] S. Faure, J. F. Bobo, J. Carrey, F. Issac, and D. Prost, "Composant sensible pour dispositif de mesure de champ électromagnétique par thermofluorescence, procédés de mesure et de fabrication correspondants," French Patent 1758907, Sep. 26, 2017.

[3] J. Vernieres, J. F. Bobo, D. Prost, F. Issac, and F. Boust, "Ferromagnetic microstructured thin films with high complex permeability for microwave applications," J. Appl. Phys., vol. 109, no. 7, p. 07A323, 2011.

[4] J. Vernieres, J. F. Bobo, D. Prost, F. Issac, and F. Boust, "Microwave magnetic field imaging using thermo-emissive ferromagnetic microstructured films," IEEE Trans. Magn., vol. 47, no. 9, pp. 2184-2187, Sep. 2011.

[5] FLEX Suppressor-Noise Suppression Sheet. May 30, 2017. [Online]. Available: https://www.tokin.com/english/product/pdf_dl/flex.pdf2184

[6] J. Carrey, B. Mehdaoui, and M. Respaud, "Simple models for dynamic hysteresis loop calculations of magnetic single-domain nanoparticles: Application to magnetic hyperthermia optimization," J. Appl. Phys., vol. 109, no. 8, 083921, 2011.

[7] T. Crépin, F. Issac, D. Prost, and S. Bolioli, "Microwave electric field imaging of metamaterials using thermoemissive films," IEEE Antennas Propag. Mag., vol. 56, no. 3, pp. 37-42, Jun. 2014. 